\title{
Assessment of Perception of Orthodontic Dental Staff about Hand Hygiene at Selected Dental Clinics, Saudi Arabia
}

\author{
Dr. Ibrahim Abdullah Alanazi ${ }^{1}$, Dr. Hussam Muneer Alqahtani ${ }^{2}$ \\ ${ }^{1}$ Dental Resident, Armed Forces Hospital, Tabuk \\ ${ }^{2}$ Dental Resident, Ministry of Health, Bishah
}

\begin{abstract}
Background: Notwithstanding the prominence on patient protection at dental clinics, some health institutes has examined the level to which wellbeing is an intentional priority or their background chains patient safety. In comeback to the Institute of Dental Medical report $n$ relation to Orthodontic subspecialty and to an executive eagerness to patient protection, the current research was applied to enhance an efficient dimension of hand hygiene. Objective: To assess the level of perception of Orthodontic dental staff about hand hygiene at the selected dental clinics, Saudi Arabia. Method: A cross-sectional survey was conducted at five dental clinics, Saudi Arabia. A18 items self-administered questionnaire was provided to 150 Orthodontic staff in the research setting based on their area of their specialties to assess the level of perception of Orthodontic dental staff about hand hygiene at the selected dental clinics, Saudi Arabia. Results: This study finding showed that the level of perception of Orthodontic dental staff about hand hygiene. The majority (95.33\%) of the Orthodontic staff had a high level of Level of Perception of Orthodontic Dental Staff about Hand Hygiene in relation to for Hand washing is indicated between tasks and procedures on the same patient. On the other hand (92.77\%) of the Orthodontic staff had a lowest level of Level of Perception of Orthodontic Dental Staff about Hand Hygiene in relation to Hand washing reduces the incidence of healthcare-related infections. As well as, (92.77\%) In standard hand washing: minimum duration should be. Although, concerning the Intermittent Level of Awareness the highest level regarding the Level of Perception of Orthodontic Dental Staff about Hand Hygiene was (2.0\%) in relation to Hand washing is indicated between tasks and procedures on the same patient. While, regarding the Low Level of Perception of Orthodontic Dental Staff about Hand Hygiene l in the item of in standard hand washing: minimum duration should be and also $n$ the item of was Alcohol hand rub substitutes hand washing even if the hands are soiled showed higher rate of awareness (4.66\%). Conclusions: The current study results revealed that there were high levels of perception of Orthodontic dental staff about hand hygiene within the study setting.
\end{abstract}

Keywords: Hand Hygiene; Perception; \& Orthodontic staff

\section{Introduction}

Progressively, dental clinics are sustaining its alertness toward the insinuation of transforming organizational culture in order to develop patient safety. Developing significance in safety culture has been accompanied by the need for appraisal utensils focused on the cultural aspects of patient protection expansion efforts. Hand Hygiene assessment tools could be utilized as a instrument for humanizing patient safety. It possibly will moreover illustrate the individuality of culture appraisal tools currently obtainable and discusses their current and potential uses, including brief examples from healthcare organizations that have commence such assessments. (Applin, Williams \& Day, 2011).

The capacity of practice in professional and ecological health care has greatly expanded with increased importance on health promotion and health fortification services. Various variables have influenced the evolution of occupational health practice. Among them are the changing population and workforce, the introduction of new chemicals and work processes into the work environment, increased work demands, technological advances and regulatory mandates, increased focus on illness/injury prevention, and a rise in health care costs and workers' compensation claims. Tiwari, Lai \& Yuen, (2006).
According to Chan.et al., (2000), suitable reporting of jobrelated exposures to an employee health service is required to ensure proper analysis, make high quality prognosis after early treatment, and establish legal prerequisites for workers' recompense. Failure to adherence to proper hand hygiene, placing dentist and patients at unnecessary risk. Information is limited regarding the prevalence of needle stick injuries, the circumstances surrounding them, and the barriers to reporting them. We conducted this study to investigate the prevalence and context of needle stick injuries and behavior associated with the reporting of injuries among a large number of dentists in training.

Hazards caused by non adherence to hand hygiene by the health care providers, statistics reported by the Central Register of Occupational Diseases in Poland indicates that among 314 new cases of occupational diseases in HCWs in 2005, HBV and HCV represented $42.6 \%$ of all cases.9 Despite the substantial reduction in $\mathrm{HBV}$ infection since vaccination was introduced in 1989, the incidence of HCV hepatitis in Poland is still on the increase in this occupational group. (Kumar, 2003).

Orthodontic staff should have a high level of awareness about occupational injury enhance consciousness education has not been prominent among health care workers, particularly in developing countries. To the greatest of our understanding, the attentiveness of Orthodontic staff in

Volume 6 Issue 1, January 2017 


\section{International Journal of Science and Research (IJSR) \\ ISSN (Online): 2319-7064 \\ Index Copernicus Value (2015): 78.96 | Impact Factor (2015): 6.391}

relation to knowledge and awareness about policies of safety measures within the work setting. Consequently, conducted this study to assess the level of perception of Orthodontic dental staff about hand hygiene within the dental clinics, Saudi Arabia.

\section{Participants and Methods}

This study was conducted in May, 2016 among Orthodontic staff at the dental clinics, Saudi Arabia. The study was granted ethical approval by the clinics ethical committee.

The participants were selected from the selected dental clinics. After signing an informed written consent form, the questionnaire was given to each participant. Before administration of the questionnaire, the purpose of the study was explained to each respondent and confidentiality of the information guaranteed.

The research was carried out by the authors who were appropriately trained in administering the informed consent and the self-report questionnaire to the participants. In this cross-sectional study, a structured questionnaire prepared by the authors, was administered to the participants.A18-item self-administered structured questionnaire about perception about hand hygiene among Orthodontic staff, which advised de novo and tested. It included a full range of response options, designed to identify the practitioner's to assess their level perception of Orthodontic dental staff about hand hygiene within the study setting.
Prior to distribution of the questionnaire, a pilot study was done on a selective group of health care workers who were asked to fill out the questionnaire and return it back with their remarks and criticism. Minor changes were then made to the final tool.

The preliminary part of the questionnaire consisted of demographic information such as occupation, age, gender, and the marital status. The second part of the questionnaire comprised of questions regarding their level of awareness' towards occupational injury among medical staff. This part also assessed awareness' towards occupational injury. It took approximately 15 minutes to complete each appraisal.

The level of perception of Orthodontic dental staff about hand hygiene within the study setting.. By examining questions. A score of " 1 " was assigned for a correct answer and " 0 " for an incorrect answer. A health care worker who obtained a total score of " 5 " was considered "very aware;" "4 or 3" "somewhat aware;" and "1 or 0" "not aware."The data were coded and analyzed by SPSS $\AA$ for Windows ${ }^{\circledR}$ ver. 12.0. Strict confidentiality was maintained. All the data were stored in computers at a secured location, with access provided only to the researchers involved in the study. The $\chi 2$ test was used to test association between categorical variables. A p value $<0.05$ (two-tailed) was considered statistically significant differences.

\section{Results}

Level of Perception of Orthodontic Dental Staff about Hand Hygiene at the Selected Clinics

\begin{tabular}{|c|c|c|c|c|c|c|}
\hline \multirow[t]{2}{*}{ Items of perception about Hand Hygiene } & \multicolumn{2}{|c|}{$\begin{array}{l}\text { High Level of } \\
\text { Awareness }\end{array}$} & \multicolumn{2}{|c|}{$\begin{array}{c}\text { Intermittent Level } \\
\text { of Awareness }\end{array}$} & \multicolumn{2}{|c|}{$\begin{array}{c}\text { Low Level of } \\
\text { Awareness }\end{array}$} \\
\hline & No & $\%$ & No & $\%$ & No & $\%$ \\
\hline Hand washing minimizes microorganisms acquired on the hands if soiled & 139 & $93.77 \%$ & 6 & $4.0 \%$ & 5 & $3.33 \%$ \\
\hline Hand washing reduces the incidence of healthcare-related infections & 138 & $92.77 \%$ & 8 & $5.33 \%$ & 3 & $2.0 \%$ \\
\hline Standard hand washing includes washing of both hands and wrists & 142 & $95.77 \%$ & 4 & $2.66 \%$ & 4 & $2.66 \%$ \\
\hline In standard hand washing: minimum duration should be & 138 & $92.77 \%$ & 5 & $3.33 \%$ & 7 & $4.66 \%$ \\
\hline Hand decontamination: includes washing the--with antiseptic soap for 30 seconds & 140 & $76.44 \%$ & 4 & $2.66 \%$ & 6 & $4.0 \%$ \\
\hline Alcohol hand rub substitutes hand washing even if the hands are soiled & 139 & $93.77 \%$ & 4 & $2.66 \%$ & 7 & $4.66 \%$ \\
\hline Hand washing is indicated between tasks and procedures on the same patient & 143 & $95.33 \%$ & 3 & $2.0 \%$ & 4 & $2.66 \%$ \\
\hline
\end{tabular}

This study finding showed that Level of Perception of Orthodontic Dental Staff about Hand Hygiene. The majority $(95.33 \%)$ of the Orthodontic staff had a high level of Level of Perception of Orthodontic Dental Staff about Hand Hygiene in relation to for Hand washing is indicated between tasks and procedures on the same patient. On the other hand $(92.77 \%)$ of the Orthodontic staff had a lowest level of Level of Perception of Orthodontic Dental Staff about Hand Hygiene in relation to Hand washing reduces the incidence of healthcare-related infections. As well as, (92.77\%) In standard hand washing: minimum duration should be. Although, concerning the Intermittent Level of Awareness the highest level regarding the Level of Perception of Orthodontic Dental Staff about Hand Hygiene was (2.0\%) in relation to Hand washing is indicated between tasks and procedures on the same patient. While, regarding the Low Level of Perception of Orthodontic Dental Staff about Hand Hygiene was in the item of in standard hand washing: minimum duration should be and also $\mathrm{n}$ the item of was Alcohol hand rub substitutes hand washing even if the hands are soiled showed higher rate of awareness (4.66\%)

\section{Discussion}

Level of Perception of Orthodontic Dental Staff about Hand Hygiene was showed significantly associated with many variables (Table 1). This study finding showed that Level of Perception of Orthodontic Dental Staff about Hand Hygiene. The majority $(95.33 \%)$ of the Orthodontic staff had a high level of Level of Perception of Orthodontic Dental Staff about Hand Hygiene in relation to for Hand washing is indicated between tasks and procedures on the same patient. On the other hand $(92.77 \%)$ of the Orthodontic staff had a lowest level of Level of Perception of Orthodontic Dental Staff about Hand Hygiene in relation to Hand washing reduces the incidence of healthcare-related infections. As 


\section{International Journal of Science and Research (IJSR) \\ ISSN (Online): 2319-7064}

Index Copernicus Value (2015): 78.96 Impact Factor (2015): 6.391

well as, (92.77\%) In standard hand washing: minimum duration should be. Although, concerning the Intermittent Level of Awareness the highest level regarding the Level of Perception of Orthodontic Dental Staff about Hand Hygiene was $(2.0 \%)$ in relation to Hand washing is indicated between tasks and procedures on the same patient. While, regarding the Low Level of Perception of Orthodontic Dental Staff about Hand Hygiene was in the item of in standard hand washing: minimum duration should be and also $n$ the item of was Alcohol hand rub substitutes hand washing even if the hands are soiled showed higher rate of awareness (4.66\%).

Another study reported by Berry,( 2008), involving 550 medical students and residents during the 1989-1990 training year likewise reported a high prevalence of hand hygiene (71\%), and a higher frequency of infection (by a factor of 6) among surgical residents than among medical residents. In these two studies, rates of reporting hand hygiene ranged from 9 to $19 \%$, and a more recent survey of all types of providers found that $34 \%$ had reported their exposure to an employee health service. On the other hand, the study carried by Klegeris \& Hurren (2011), showed that only $49 \%$ of surgical residents report such injuries extends previous observations that underreporting may result in a substantial underestimation of the magnitude of the problem of non adherence of hand hygiene.

Concerning the Intermittent Level of Awareness the highest level was awareness towards Perception of Orthodontic Dental Staff about Hand Hygiene in relation to Hand washing Alcohol hand rub substitutes hand washing even if the hands are soiled showed higher rate of awareness (4.66\%).It is very important that health care workers have good understanding about the risk of blood-borne pathogens at work place and about the preventive measures for reducing risk. In this study, the majorities of the respondents were very knowledgeable of the harmful effects of awareness of dentists universal precautions compared with those who served for shorter periods. Training and education have been found to be of paramount importance to developing awareness among health care workers, as well as improving adherence to high-quality clinical practice. This research findings is congruent with the findings of the study carried out by (Frier,2011), who found that he greater awareness of hand hygiene among health care workers employed for a longer period non-compliance among medical doctors and nurses are associated with insufficient knowledge, workload, forgetfulness, workplace safety .

Hand Hygiene reduces the risk of exposure of the health care provider's skin or mucous membranes to potentially infectious materials. Protective barriers reduce the risk of exposure to blood and other body fluids to which universal precautions apply. Examples of protective barriers include gloves, gowns, masks, and protective eyewear. Just over one half of the respondents indicated that they were provided with protective equipment most times. Furthermore, more nurses were provided with protective equipment than medical technologists and medical doctors. Interestingly, more respondents who were aware of universal precautions reported being provided with protective equipment more often than those who were somewhat or not aware. This study results congruent with the research data carried out by Dane,(2013), who reported that less than two-thirds of health care workers claimed that they always used personal protective equipment such as aprons, gowns and gloves, during surgeries and while conducting deliveries. According to Jawaid, et al, among medical doctors working in a tertiary care hospital in Pakistan, compliance for hand washing was $86 \%$, for wearing gloves was $79 \%$, masks $46 \%$, eye goggles $25 \%$ and for using gowns/plastic aprons was $45 \% .35$ However, according to Kwan (2012), there is sometimes a high rate of non-compliance among health care workers and this may be due to a lack of understanding among health care workers of how to properly use protective barriers.

\section{Acknowledgements}

Appreciation is hereby extended to all the participants and administrators staff at study setting

\section{References}

[1] Applin H, Williams B, Day R, Buro K. A comparison of competencies between problem-based learning \& nonproblem-based graduate nurses. Nurse Educ Today 2011; 31: 129-34

[2] Tiwari A, Lai P, So M, Yuen K. A comparison of the effects of problem-based learning and lecturing on the development of students' critical thinking, Med Edu 2006; 40: 547-54.

[3] Chan JNF, Chan CPK, Chan P, Chan LP. Undergraduate medical education: comparison of problem-based learning and conventional teaching. Hong Kong Med J 2000; 6: 301-6.

[4] Kumar S. An innovative method to enhance interaction during lecture sessions. Adva Physiol Educ 2003; 25: 20-5.

[5] Berry W. Surviving lecture: a pedagogical alternative. College Teach 2008; 56: 102-6.

[6] Klegeris A, Hurren H. Impact of problem-based learning in a large classroom setting: student perception and problem-solving skills. Adv Physiol Educ 2011; 35: 408-15.

[7] Frier BM. Cognitive functioning in type 1 diabetes: the Diabetes Control and Complications Trial (DCCT) revisited. Diabetologia 2011; 54: 233-6

[8] Clason DL, Dormody TJ. Analyzing Data Measured by Individual Likert-Type Items. J Agricultural Educ 1994, 35: $31-5$

[9] Dane Bertram. Likert Scale are the meaning of life. CPSC 681-Topic Report. [online] 2013 [2013 Nov 21]. Available from: URL: http://poincare.matf.bg.ac.rs/ kristina/topic-danelikert.pdf

[10]Blumberg P, Eckenfels E. A comparison of student satisfaction with their preclinical environment in a traditional and a problem based curriculum. Res Med Educ 1988; 27: 60-5.

[11] Kaufman DM, Mann KV. Comparing student's attitudes in problem based and conventional curricula. Acad Med 1996; 71: 1096-9.

\section{Volume 6 Issue 1, January 2017


[12] Kwan CY. Dental and medical students' perspectives on early exposure to PBL in Taiwan. J Dent Educ 2012; 76: 746-51.

[13] Anderson V, Reid K. Students' perception of a problembased learning scenario in dental nurse education. Eur J Dent Educ 2012; 16: 218-23.

[14] Hwang SY, Kim MJ. A comparison of problem-based learning and lecture-based learning in an adult health nursing course. Nurse Educ Today 2006; 26: 315-21.

[15] McParland M, Noble LM, Livingston G. The effectiveness of problem-based learning compared to traditional teaching in undergraduate psychiatry. Med Edu 2004; 38: 859-67.

[16] Sahin M, Yorek N. A comparison of problem-based learning and traditional lecture students' expectations and course grades in an introductory physics classroom. Sci Res Essay 2009; 4: 753-62.

[17]Dehkordi AH, Heydarnejad MS. The impact of problem-based learning and ecturing on the behavior and attitudes of Iranian nursing students. A randomised controlled trial. Dan Med Bull 2008; 55: 224-6.

[18] Beachey WD. A Comparison of Problem-Based Learning and Traditional Curricula in Baccalaureate Respiratory Therapy Education. Respir Care 2007; 52: 1497-506.

[19]Hwang SY, Kim MJ. A comparison of problem-based learning and lecture-based learning in an adult health nursing course. Nurse Educ Today 2006; 26: 315-21.

[20] Kim AR, Kim YK, Song YS, Shin KR, Ahn HJ, Lee JS, et al. A study for the development of a problem based learning package for patients with perceptionadjustment disorder. J Korean Adult Health Nurs 2001; 13: 385-96.

[21] Kim SA, Kang IA, Kim S, Nam KA, Park JH. Development of a problem-based learning program in nursing education curriculum. J Korean Psychiatr Nurs 2000; 9: 559-70.

[22] Rideout E, England-Oxford V, Brown B, FothergillBourbonnais $\mathrm{F}$, Ingram $\mathrm{C}$, Benson $\mathrm{G}$, et al. A comparison of problem-based and conventional curricula in nursing education. Adv Health Sci Educ Theory Pract 2007; 7: 3-17.

[23] Habib F, Baig L, Mansuri FA. Opinion of Medical Students regarding Problem Based Learning. J Pak Med Assoc 2006; 56: 430-2.

[24] Usmani A, Sultan ST, Ali S, Fatima N, Babar S. Comparison of students and facilitators perception of implementing problem based learning. J Pak Med Assoc 2011; 61: 332-5.

[25] Baig LA, Asad F. Introducing problem-based learning in a medical school with traditional/conventional curriculum. J Coll Physicians Surg Pak 2003; 13: 37881 . 